\title{
Chemical Control of Morning Glory as a Function of Water RESTRICTION LEVELS ${ }^{1}$
}

\author{
Controle Químico de Cordas-de-Viola em Função de Níveis de Restrição Hídrica
}

\author{
GIANCOTTI, P.R.F. ${ }^{2}$, TOLEDO, R.E.B. ${ }^{3}$, ALVES, P.L.C.A. ${ }^{2}$, VICTORIA FILHO, R. ${ }^{4}$, CASON, J.B. ${ }^{3}$, \\ and ROCHA, M.G. ${ }^{3}$
}

\begin{abstract}
Among the herbicides recommended for the dry season and registered to sugarcane crop, amicarbazone, isoxaflutole and the association diuron + hexazinone + sulfomethuron-methyl can be highlighted. These are pre-emergence herbicides efficient against broad-leaved weeds. Morning glory causes large losses in infested sugarcane fields by bending the stalks and interfering in harvesting. In this study the effectiveness of preemergence herbicides for two species of morning glory (Ipomoea hederifolia and Ipomoea grandifolia) was evaluated. Treatments were arranged in completely randomized factorial design (4 x 7). There were four periods of water restriction (0,30, 60 and 90 days), seven chemical treatments [diuron + hexazinone + sulfometuron-methyl $(1387+391+33.35 \mathrm{~g}$ a.i. ha-1 $)$, diuron + hexazinone + sulfometuron-methyl $\left(1507.5+425+36.25 \mathrm{~g}\right.$ a.i. ha $\left.{ }^{-1}\right)$, diuron + hexazinone + sulfometuron-methyl $(1658.25+467.5+39.87 \mathrm{~g}$ a.i. ha-1), diuron + hexazinone + sulfometuronmethyl $\left(1809+510+43.5 \mathrm{~g}\right.$ a.i. $\left.\mathrm{ha}^{-1}\right)$, amicarbazone $\left(1190 \mathrm{~g}\right.$ a.i. ha $\left.{ }^{-1}\right)$, amicarbazone + isoxaflutole $\left(840+82.5 \mathrm{~g}\right.$ a.i. $\left.\left.\mathrm{ha}^{-1}\right)\right]$ and a control with no application. At 7, 14, 21 and 28 days after the restoration of moisture, control was visually evaluated. After the final evaluation, the dry mass of morning glories was measured. At 90 days of water restriction, diuron + hexazinone + sulfometuron-methyl was more effective to control $I$. hederifolia than the amicarbazone + isoxaflutole tank mixture. The four diuron + hexazinone + sulfometuronmethyl doses have reduced morning glory dry mass to zero; whereas treatments with amicarbazone have not. The most effective treatment for morning glory control was diuron + hexazinone + sulfometuron-methyl. This result may be due to a possible synergistic interaction.
\end{abstract}

Keywords: chemical control, Ipomoea hederifolia, Ipomoea grandifolia, weed science.

\begin{abstract}
RESUMO - Entre os herbicidas recomendados para a época seca e registrados para a cultura da cana-de-açúcar estão amicarbazone, isoxaflutole e a associação diuron + hexazinona + sulfometuronmetil. Esses herbicidas apresentam ação em pré-emergência e atuam em plantas daninhas de folha larga. Cordas-de-viola provocam grandes perdas em canaviais infestados, causando tombamento da cana e interferindo nas operações de colheita. Neste estudo foi avaliada a eficácia de controle de herbicidas pré-emergentes em duas espécies de corda-de-viola (Ipomoea hederifolia e Ipomoea grandifolia). Os tratamentos foram dispostos em esquema fatorial $4 \times 7$. Foram quatro periodos de restrição hidrica $(0,30,60$ e 90 dias), sete tratamentos quimicos [diuron + hexazinona + sulfometuron-metil $\left(1.387+391+33,35 \mathrm{~g}\right.$ i.a. $\left.\mathrm{ha}^{-1}\right)$, diuron + hexazinona + sulfometuron-metil (1.507,5 $+425+36,25 \mathrm{~g}$ i.a. $\left.\mathrm{ha}^{-1}\right)$, diuron + hexazinona + sulfometuron-metil $(1.658,25+467,5+$ 39,87 g i.a. ha $\left.\mathrm{g}^{-1}\right)$, diuron + hexazinona + sulfometuron-metil $\left(1.809+510+43,5 \mathrm{~g}\right.$ i.a. $\left.\mathrm{ha}^{-1}\right)$, amicarbazone (1.190 g i.a. ha $^{-1}$ ) e amicarbazone + isoxaflutole $(840+82,5 \mathrm{~g}$ i.a. ha-1)] e um controle sem aplicação. Após 7, 14, 21 e 28 dias do restabelecimento da umidade, foram realizadas avaliações visuais de controle. Depois da última avaliação, foi determinada a massa seca das cordas-de-viola. Após 90 dias de restrição hidrica, diuron + hexazinona + sulfometuron-metil controlou I. hederifolia de forma mais eficaz que a mistura de amicarbazone + isoxaflutole. As quatro doses de diuron +
\end{abstract}

Recebido para publicação em 5.9.2013 e aprovado em 20.1.2014.

2 UNESP, Jaboticabal-SP, Brasil, <paulogiancotti@gmail.com>; ${ }^{3}$ DuPont, Barueri-SP, Brasil; ${ }^{4}$ Escola Superior Luís de Queiros, Piracicaba-SP, Brasil.

Planta Daninha, Viçosa-MG, v. 32, n. 2, p. 345-353, 2014 
hexazinona + sulfometuron-metil reduziram a massa seca das cordas-de-viola a zero, o que não aconteceu com os tratamentos com amicarbazone. O tratamento mais eficaz para o controle de cordade-viola foi diuron + hexazinona + sulfometuron-metil em todas as doses estudadas; esse resultado pode se dever a possiveis relações de sinergismo entre esses produtos.

Palavras-chave: controle químico, Ipomoea hederifolia, Ipomoea grandifolia, matologia.

\section{INTRODUCTION}

Weed control by tillage may benefit the sugarcane field by aerating and improving the physical conditions of soil, but it is probably more often deleterious by adversely affecting soil structure, damaging foliage and root pruning. Thus, herbicides and reduced cultivation may become more important for weed control in sugarcane as cost of fuel increases (Chitsaz \& Nelson, 1983).

The success of chemical control depend on soil type, availability of soil moisture, and species selection (Schroeder, 1988). Previous work has shown that control with preemergence herbicides is possible, but seed germinating at depth, or in dry or cloddy soils, are not controlled as effectively (Garrett \& Orson, 1989). Furthermore, the persistence of herbicides in the soil varies according to the chemical structure of molecule, soil type, and climatic conditions, such as moisture, which affects adsorption, lixiviation and microbial and chemical decomposition (Silva et al., 1999). Herbicides require moisture in soil for its molecules to become available in the soil solution and then eventually be absorbed by weeds (Martini \& Durigan, 2004). Chemical control is more effective if spraying is conducted during the rainy season, due to soil moisture and the intense metabolism of weeds, which helps in the absorption of most herbicides authorized for sugarcane crop, since they are highly soluble in water (Rodrigues \& Almeida, 2011). However, there are also products on the market that are also efficient during the dry season. In recent studies Negrisoli et al. (2011) and Toledo et al. (2010) documented great weed control with amicarbazone, isoxaflutole, diuron and hexazinone, due to favorable physical and chemical characteristics like high water solubility and low adsorption capacity of clay particles and organic matter.
The herbicides recommended for the dry season and authorized for sugarcane, include amicarbazone, isoxaflutole and the association diuron + hexazinone + sulfomethuron-methyl. These herbicides have action at preemergence and act against both grasses and broad-leaved weeds.

Amicarbazone is a triazolinone herbicide with a broad spectrum of weed control; it acts by inhibiting the photosystem II. The phenotypic responses of sensitive plants exposed to amicarbazone include chlorosis, stunted growth, tissue necrosis, and death (Toledo et al., 2004; Dayan et al., 2009). Isoxaflutole is a sugarcane herbicide that causes characteristic bleaching of newly developed tissues of susceptible species followed by growth paralysis and necrosis (Luscombe et al., 1995; Pallet et al., 1998). This herbicide has low solubility in water, is stable at drought, and has a residual effect which may exceed 60 days after the outset of rains (Beltran et al., 2001; Rodrigues \& Almeida, 2011).

Among the molecules used in agriculture, diuron is widely used as herbicide because of its inhibition of photosynthesis (Liu, 2010). Diuron is a non-ionic herbicide with intermediate water solubility of $42 \mathrm{~mL} \mathrm{~g}^{-1}$ (Hornsby et al., 1990). Degradation of phenylurea herbicides in nature can be a relatively slow process. These pesticides can be decomposed by UV irradiation or by acidic or alkaline conditions. Biological degradation of the compounds in plants and soil is carried out by microflora and microfauna (Liu, 2010). Hexazinone is another inhibitor of photosynthesis, and it is a broad-spectrum contact herbicide used in a variety of crop and non-crop applications. It is highly soluble in water and mobile in soil. Hexazinone may be absorbed through both roots and leaves, and acts as an inhibitor of photosynthesis 
(Peterson et al., 1997). Sulfometuron-methyl is a sulfonylurea, effective in controlling many annual and perennial grasses and broadleaved weeds in non-cropland areas and sites with plants. Decomposition rate of the active ingredient sulfometuron-methyl is fast under field conditions (Zahnow, 1985). The pKa of sulfometuron is 5.7 and its solubility in water at $25^{\circ} \mathrm{C}$ is $10 \mathrm{ppm}$ at pH 5 and $300 \mathrm{ppm}$ at pH 7 . By thin-layer chromatography, Wehtje et al. (1987) showed that sulfometuron is slightly more mobile and more subjected to sorption than imazapyr.

Diuron, hexazinone and sulfometuronmethyl are available for sugarcane producers as mixed commercial herbicides. Producers prefer tank mixtures because they reduce application costs and potentially increase the spectrum of controlled weeds. However, both synergistic and antagonistic interactions can occur with tank mixtures (Hydrik \& Shaw, 1994).

Thaker \& Singh (1954) reported that morning glory caused 20 to $25 \%$ losses in infested sugarcane fields by twining around clumps, bending stalks, damaging tops, causing stalks to remain undeveloped and interfering with harvest operations. Eastman \& Coble (1977) observed that morning glories were resistant to commonly used preemergence herbicides in North Carolina, USA. As dominant weeds, morning glories cause increased lodging, reduced yields, and reduced efficiency of mechanical harvest (Yonce \& Palmer, 1976).

The association of active ingredients with different water restriction characteristics may provide a better control of morning glory weeds through synergism action. The aim of this study was to determine the most effective chemical treatment for two species of "morning glory" at increasing periods of water restriction.

\section{MATERIALS AND METHODS}

The experiment was conducted in 2011, at greenhouse, in the Department of Biology Applied to Agriculture, FCAV/UNESP, Jaboticabal. Two species of morning glory were sown in $5 \mathrm{~L}$ pots filled with clay soil. Oxisoil was collected at a top layer soil in an agricultural area of Jaboticabal, Brazil. Each pot was a plot and in it $3 \mathrm{~g}$ of Ipomoea hederifolia, Ipomoea grandifolia were sown. The treatments were arranged in completely randomized factorial design $(4 \times 7)$, where factor A included four periods of water restriction and, factor $B$, seven chemical treatments, with three replications. Application of the following chemical treatments was carried out at pre-emergence: diuron + hexazinone + sulfometuron-methyl $\left(1387+391+33.35\right.$ g a.i. ha $\left.{ }^{-1}\right)$, diuron + hexazinone + sulfometuron-methyl $(1507.5+$ $425+36.25$ g a.i. ha $^{-1}$ ), diuron + hexazinone + sulfometuron-methyl $(1658.25+467.5+$ $39.87 \mathrm{~g}$ a.i. $\mathrm{ha}^{-1}$ ), diuron + hexazinone + sulfometuron-methyl $(1809+510+$ $43.5 \mathrm{~g}$ a.i. ha-1), amicarbazone (1190 g a.i. ha-1), amicarbazone + isoxaflutole $(840+$ $82.5 \mathrm{~g}$ a.i. $\mathrm{ha}^{-1}$ ) and a control with no application. Herbicide application was performed using a $\mathrm{CO}_{2}$ pressurized backpack sprayer adjusted to spread a volume of $200 \mathrm{~L} \mathrm{ha}^{-1}$. After application, pots were subjected to $0,30,60$ and 90 days of water restriction (DWR) inside a greenhouse, protected from rain. After the periods of water restriction, the pots were watered daily.

At $7,14,21$ and 28 days after the restoration of moisture (DARM), control was visually evaluated by using a scale ranging from 0 to $100 \%$, with 0 meaning no injury, and $100 \%$, plant death. After the final evaluation (28 DARM), plants were cut at ground level, placed on paper bags and dried inside forced air oven, at a temperature of $70{ }^{\circ} \mathrm{C}$, until the sample reached constant dry mass weight. The material was weighed at $0.0001 \mathrm{~g}$ digital scale accuracy.

Data found were subjected to variance analysis (F-test), and then submitted to Tukey test at $5 \%$ probability to compare means.

\section{RESULTS AND DISCUSSION}

There was interaction between DWR and chemical treatments only in the last evaluation (Table 1). At 7 DARM, the control of I. grandifolia was higher with 90 DWR (Table 1). At 14 and 21 DARM, no differences were found for DWR. On the last evaluation, 
weeds treated with no water restriction were fully controlled. Furthermore, longer period of drought increased weed control.
No differences were found among treatments for control efficiency. However, the mixture diuron + hexazinone + sulfometuron-

Table 1 - Mean visual assessment of I. grandifolia control as a function of days of water restriction after application (DWR) and chemical treatments. Visual evaluation was carried out at four different days after the restoration of moisture (DARM)

\begin{tabular}{|c|c|c|c|c|}
\hline DWR & 7 DARM & 14 DARM & 21 DARM & 28 DARM \\
\hline 0 & $48.61 \mathrm{~b}^{\frac{1 /}{}}$ & $99.22 \mathrm{a}$ & $97.06 \mathrm{a}$ & $100.00 \mathrm{a}$ \\
\hline 30 & $58.33 \mathrm{~b}$ & $97.61 \mathrm{a}$ & $99.72 \mathrm{a}$ & $99.83 \mathrm{ab}$ \\
\hline 60 & $59.17 \mathrm{~b}$ & $98.06 \mathrm{a}$ & $99.56 \mathrm{a}$ & $99.17 \mathrm{ab}$ \\
\hline 90 & $92.97 \mathrm{a}$ & $97.92 \mathrm{a}$ & $99.44 \mathrm{a}$ & $98.06 \mathrm{~b}$ \\
\hline \multicolumn{5}{|l|}{ Chemical treatment } \\
\hline Diu+Hex+Sulfom & $62.12 \mathrm{a}$ & $99.92 \mathrm{a}$ & $100.00 \mathrm{a}$ & $100.00 \mathrm{a}$ \\
\hline Diu+Hex+Sulfom & $73.92 \mathrm{a}$ & $96.25 \mathrm{a}$ & $97.67 \mathrm{a}$ & $100.00 \mathrm{a}$ \\
\hline Diu+Hex+Sulfom & $59.75 \mathrm{a}$ & $98.54 \mathrm{a}$ & $98.75 \mathrm{a}$ & $100.00 \mathrm{a}$ \\
\hline Diu+Hex+Sulfom & $60.21 \mathrm{a}$ & $98.75 \mathrm{a}$ & $98.75 \mathrm{a}$ & $99.17 \mathrm{a}$ \\
\hline Amicarbazone & $62.79 \mathrm{a}$ & $100.00 \mathrm{a}$ & $99.75 \mathrm{a}$ & $97.92 \mathrm{a}$ \\
\hline Amicarb+Isoxafl & $69.83 \mathrm{a}$ & $95.75 \mathrm{a}$ & $98.75 \mathrm{a}$ & $98.50 \mathrm{a}$ \\
\hline $\mathrm{CV}(\%)$ & 33.28 & 5.81 & 3.86 & 1.82 \\
\hline F treat. & $0.86^{\mathrm{ns}}$ & $1.21^{\mathrm{ns}}$ & $0.58^{\mathrm{ns}}$ & $2.98^{\mathrm{ns}}$ \\
\hline F drought & $14.58 * *$ & $0.27^{\mathrm{ns}}$ & $1.98^{\mathrm{ns}}$ & $4.31 * *$ \\
\hline F treat.xdrought & $1.73^{\text {ns }}$ & $0.73^{\text {ns }}$ & $0.64^{\mathrm{ns}}$ & $2.53 * *$ \\
\hline LSD treat. & 20.55 & 6.91 & 4.62 & 2.18 \\
\hline LSD drought & 28.03 & 5.06 & 3.39 & 1.60 \\
\hline
\end{tabular}

** Significant at $\alpha=0.01$. ns Not significant at $\alpha=0.05$. ${ }^{1 /}$ Within a column, means followed by the same letter are not significantly different according to Tukey's Multiple Mean Comparison test.

Table 2 - Visual assessment of I. grandifolia control as a function of days of water restriction after application (DWR) and chemical treatments. Visual evaluation was carried out at four different days after the restoration of moisture (DARM)

\begin{tabular}{|c|c|c|c|c|c|c|c|c|c|}
\hline \multirow{2}{*}{ Chemical treatment } & \multirow{2}{*}{ Dose $\left(\mathrm{g} \mathrm{ha}^{-1}\right)$} & \multicolumn{4}{|c|}{ Chemical control of IAQGR at 7 DARM } & \multicolumn{4}{|c|}{ Chemical control of IAQGR at 14 DARM } \\
\hline & & 0 DWR & 30 DWR & $60 \mathrm{DWR}$ & 90 DWR & 0 DWR & 30 DWR & $60 \mathrm{DWR}$ & 90 DWR \\
\hline Diu+Hex+Sulfom & $387+391+33,35$ & $38.33 \mathrm{Ab}^{1 /}$ & 68.33 Aab & $43.33 \mathrm{Ab}$ & $98.50 \mathrm{Aa}$ & $100.00 \mathrm{Aa}$ & $99.67 \mathrm{Aa}$ & $100.00 \mathrm{Aa}$ & $100.00 \mathrm{Aa}$ \\
\hline Diu+Hex+Sulfom & $1507,5+425+36,25$ & $80.00 \mathrm{Aa}$ & $58.33 \mathrm{Aa}$ & $63.33 \mathrm{Aa}$ & $94.00 \mathrm{Aa}$ & $96.67 \mathrm{Aa}$ & $96.67 \mathrm{Aa}$ & $91.67 \mathrm{Aa}$ & $100.00 \mathrm{Aa}$ \\
\hline Diu+Hex+Sulfom & $1658,25+467,5+39,87$ & $43.33 \mathrm{Ab}$ & $61.67 \mathrm{Aab}$ & $35.00 \mathrm{Ab}$ & $99.00 \mathrm{Aa}$ & $100.00 \mathrm{Aa}$ & $96.67 \mathrm{Aa}$ & $100.00 \mathrm{Aa}$ & $97.50 \mathrm{Aa}$ \\
\hline Diu+Hex+Sulfom & $1809+510+43,5$ & $53.33 \mathrm{Aa}$ & $53.33 \mathrm{Aa}$ & $56.67 \mathrm{Aa}$ & $77.50 \mathrm{Aa}$ & $98.67 \mathrm{Aa}$ & $99.67 \mathrm{Aa}$ & $96.67 \mathrm{Aa}$ & $100.00 \mathrm{Aa}$ \\
\hline Amicarbazone & 1190 & $33.33 \mathrm{Ab}$ & $31.67 \mathrm{Ab}$ & $86.67 \mathrm{Aa}$ & $99.50 \mathrm{Aa}$ & $100.00 \mathrm{Aa}$ & $100.00 \mathrm{Aa}$ & $100.00 \mathrm{Aa}$ & $100.00 \mathrm{Aa}$ \\
\hline Amicarb+Isoxafl & $840+82,5$ & $43.33 \mathrm{Aa}$ & $76.67 \mathrm{Aa}$ & $70.00 \mathrm{Aa}$ & $89.33 \mathrm{Aa}$ & $100.00 \mathrm{Aa}$ & $93.00 \mathrm{Aa}$ & $100.00 \mathrm{Aa}$ & $90.00 \mathrm{Aa}$ \\
\hline \multirow{2}{*}{ Chemical treatment } & \multirow{2}{*}{ Dose $\left(g h a^{-1}\right)$} & \multicolumn{4}{|c|}{ Chemical control of IAQGR at 21 DARM } & \multicolumn{4}{|c|}{ Chemical control of IAQGR at 28 DARM } \\
\hline & & $0 \mathrm{DWR}$ & $30 \mathrm{DWR}$ & $60 \mathrm{DWR}$ & $90 \mathrm{DWR}$ & 0 DWR & $30 \mathrm{DWR}$ & $60 \mathrm{DWR}$ & $90 \mathrm{DWR}$ \\
\hline Diu+Hex+Sulfom & $387+391+33,35$ & $100.00 \mathrm{Aa}$ & $100.00 \mathrm{Aa}$ & $100.00 \mathrm{Aa}$ & $100.00 \mathrm{Aa}$ & $100.00 \mathrm{Aa}$ & $100.00 \mathrm{Aa}$ & $100.00 \mathrm{Aa}$ & $100.00 \mathrm{Aa}$ \\
\hline Diu + Hex+Sulfom & $1507,5+425+36,25$ & $93.33 \mathrm{Aa}$ & $100.00 \mathrm{Aa}$ & $97.33 \mathrm{Aa}$ & $100.00 \mathrm{Aa}$ & $100.00 \mathrm{Aa}$ & $100.00 \mathrm{Aa}$ & $100.00 \mathrm{Aa}$ & $100.00 \mathrm{Aa}$ \\
\hline Diu+Hex+Sulfom & $1658,25+467,5+39,87$ & $95.00 \mathrm{Aa}$ & $100.00 \mathrm{Aa}$ & $100.00 \mathrm{Aa}$ & $100.00 \mathrm{Aa}$ & $100.00 \mathrm{Aa}$ & $100.00 \mathrm{Aa}$ & $100.00 \mathrm{Aa}$ & $100.00 \mathrm{Aa}$ \\
\hline Diu+Hex+Sulfom & $1809+510+43,5$ & $95.00 \mathrm{Aa}$ & $100.00 \mathrm{Aa}$ & $100.00 \mathrm{Aa}$ & $100.00 \mathrm{Aa}$ & $100.00 \mathrm{Aa}$ & $100.00 \mathrm{Aa}$ & $96.67 \mathrm{Aa}$ & $100.00 \mathrm{Aa}$ \\
\hline Amicarbazone & 1190 & $99.00 \mathrm{Aa}$ & $100.00 \mathrm{Aa}$ & $100.00 \mathrm{Aa}$ & $100.00 \mathrm{Aa}$ & $100.00 \mathrm{Aa}$ & $100.00 \mathrm{Aa}$ & $98.33 \mathrm{Aa}$ & $93.33 \mathrm{Bb}$ \\
\hline Amicarb+Isoxafl & $840+82,5$ & $100.00 \mathrm{Aa}$ & $98.33 \mathrm{Aa}$ & $100.00 \mathrm{Aa}$ & $96.67 \mathrm{Aa}$ & $100.00 \mathrm{Aa}$ & $99.00 \mathrm{Aa}$ & $100.00 \mathrm{Aa}$ & $95.00 \mathrm{Bb}$ \\
\hline
\end{tabular}

${ }^{1 /}$ Means within a column followed by the same uppercase letter or means within a row followed by the same lowercase letter are not significantly different according to Tukey's F-protected test at $\mathrm{P}=0.05$. 
methyl has fully controlled weeds at three doses; and the treatments with amicarbazone showed a lower control value than all doses of the mixture diuron + hexazinone + sulfometuron-methyl.

No differences were found among chemical treatments except for 90 DWR, between diuron + hexazinone + sulfometuron-methyl and amicarbazone on the last evaluation

Table 3 - I. grandifolia dry mass average as a function of days of water restriction after application (DWR) and chemical treatments. This evaluation was conducted 28 days after the restoration of moisture (DARM)

\begin{tabular}{|l|c|c|}
\hline & \multicolumn{1}{|c|}{ DWR } & $\begin{array}{c}\text { IAQGR dry mass } \\
(\mathrm{g})\end{array}$ \\
\hline & 0 & $0.0054 \mathrm{c}^{\mathrm{l}}$ \\
\hline & 30 & $0.0518 \mathrm{~b}$ \\
\hline & 60 & $0.0742 \mathrm{ab}$ \\
\hline & 90 & $0.0996 \mathrm{a}$ \\
\hline Chemical treatment & ${\text { Dose }\left(\mathrm{g} \mathrm{ha}^{-1}\right)}^{-1}$ & \\
\hline Diu+Hex+Sulfom & $387+391+33,35$ & $0.0000 \mathrm{~b}$ \\
\hline Diu+Hex+Sulfom & $1507,5+425+36,25$ & $0.0000 \mathrm{~b}$ \\
\hline Diu+Hex+Sulfom & $1658,25+467,5+39,87$ & $0.0000 \mathrm{~b}$ \\
\hline Diu+Hex+Sulfom & $1809+510+43,5$ & $0.0017 \mathrm{~b}$ \\
\hline Amicarbazone & \multicolumn{1}{|c|}{1190} & $0.0144 \mathrm{~b}$ \\
\hline Amicarb+Isoxafl & $840+82,5$ & $0.0027 \mathrm{~b}$ \\
\hline Control & \multicolumn{1}{|c|}{-} & $0.3856 \mathrm{a}$ \\
\hline & CV(\%) & 90.31 \\
\hline & F treat. & $92.25^{* *}$ \\
\hline & F drought & $12.34 * *$ \\
\hline & F treat.xdrought & $11.40^{* *}$ \\
\hline & LSD treat. & 0.0651 \\
\hline & LSD drought & 0.0426 \\
\hline
\end{tabular}

** Significant at $\alpha=0.01 .{ }^{1 /}$ Within a column, means followed by the same letter are not significantly different according to Tukey's Multiple Mean Comparison test.
(28 DARM) (Table 2). All doses of diuron + hexazinone + sulfometuron-methyl have fully controlled I. grandifolia, as opposed to other treatments.

At 28 DARM, plant dry mass increased as DWR increased. Also, 90 DWR differed from 30, and 30 differed from no water restriction (Table 3). Chemical treatments did not differ among them; they differed, however, from control.

For I. grandifolia dry mass there was interaction between the factors and DWR ranged within the control (Table 4). Plants grew more after a period of 60 DWR. All plants died under treatments with diuron + hexazinone + sulfometuron-methyl, as opposed to those of treatments with amicarbazone and amicarbazone + isoxaflutole. Nevertheless, no differences were found between these chemical treatments.

Mean visual assessment of I. hederifolia control did not differ among treatments (Table 5). Except for 7 DARM where mean DWR varied. Control at 90 DWR was greater than 30 and 0 DWR.

There was interaction between DWR and the chemical treatments for I. hederifolia control at 28 DARM (Table 5). Within 90 DWR, diuron + hexazinone + sulfometuron-methyl had fully controlled I. hederifolia and the tank mixture amicarbazone + isoxaflutole had reached a 98.33\% control (Table 6).

I. hederifolia dry mass did not differ for DWR at 28 DARM (Table 7). However it differed for

Table 4 - I. grandifolia dry mass as a function of days of water restriction after application (DWR) and chemical treatments. This evaluation was conducted 28 days after the restoration of moisture (DARM)

\begin{tabular}{|l|c|c|c|c|c|}
\hline \multirow{2}{*}{ Chemical treatment } & \multirow{2}{*}{ Dose $\left(\mathrm{g} \mathrm{ha}^{-1}\right)$} & \multicolumn{4}{c|}{ IAQGR dry mass $(\mathrm{g})$} \\
\cline { 3 - 6 } & & $0 \mathrm{DWR}$ & $30 \mathrm{DWR}$ & $60 \mathrm{DWR}$ & $90 \mathrm{DWR}$ \\
\hline Diu+Hex+Sulfom & $387+391+33,35$ & $0.0000 \mathrm{Ba}{ }^{1 /}$ & $0.000 \mathrm{Ba}$ & $0.000 \mathrm{Ba}$ & $0.0000 \mathrm{Ba}$ \\
\hline Diu+Hex+Sulfom & $1507,5+425+36,25$ & $0.0000 \mathrm{Ba}$ & $0.000 \mathrm{Ba}$ & $0.000 \mathrm{Ba}$ & $0.0000 \mathrm{Ba}$ \\
\hline Diu+Hex+Sulfom & $1658,25+467,5+39,87$ & $0.0000 \mathrm{Ba}$ & $0.000 \mathrm{Ba}$ & $0.000 \mathrm{Ba}$ & $0.0000 \mathrm{Ba}$ \\
\hline Diu+Hex+Sulfom & $1809+510+43,5$ & $0.0000 \mathrm{Ba}$ & $0.000 \mathrm{Ba}$ & $0.0067 \mathrm{Ba}$ & $0.0000 \mathrm{Ba}$ \\
\hline Amicarbazone & 1190 & $0.0000 \mathrm{Ba}$ & $0.000 \mathrm{Ba}$ & $0.0026 \mathrm{Ba}$ & $0.0550 \mathrm{Ba}$ \\
\hline Amicarb+Isoxafl & $840+82,5$ & $0.0000 \mathrm{Ba}$ & $0.000 \mathrm{Ba}$ & $0.0000 \mathrm{Ba}$ & $0.0108 \mathrm{Ba}$ \\
\hline Control & - & $0.0379 \mathrm{Ac}$ & $0.3626 \mathrm{Ab}$ & $0.6881 \mathrm{Aa}$ & $0.4539 \mathrm{Ab}$ \\
\hline
\end{tabular}

1/ Means within a column followed by the same uppercase letter or means within a row followed by the same lowercase letter are not significantly different according to Tukey's F-protected test at $\mathrm{P}=0.05$. 
Table 5 - Visual assessment average of I. hederifolia control as a function of days of water restriction after application (DWR) and chemical treatments. Visual evaluation was carried out at four different days after the restoration of moisture (DARM)

\begin{tabular}{|c|c|c|c|c|}
\hline DWR & 7 DARM & 14 DARM & 21 DARM & 28 DARM \\
\hline 0 & $66.39 \mathrm{~b}^{1 /}$ & $94.72 \mathrm{a}$ & $99.44 \mathrm{a}$ & $100.00 \mathrm{a}$ \\
\hline 30 & $70.28 \mathrm{~b}$ & $98.44 \mathrm{a}$ & $99.72 \mathrm{a}$ & $100.00 \mathrm{a}$ \\
\hline 60 & $79.50 \mathrm{ab}$ & $100.00 \mathrm{a}$ & $100.00 \mathrm{a}$ & $98.33 \mathrm{a}$ \\
\hline 90 & $93.19 \mathrm{a}$ & $100.00 \mathrm{a}$ & $100.00 \mathrm{a}$ & $99.72 \mathrm{a}$ \\
\hline \multicolumn{5}{|l|}{ Chemical treatment } \\
\hline Diu+Hex+Sulfom & $82.33 \mathrm{a}$ & $98.75 \mathrm{a}$ & $99.16 \mathrm{a}$ & $100.00 \mathrm{a}$ \\
\hline Diu+Hex+Sulfom & $71.75 \mathrm{a}$ & $99.50 \mathrm{a}$ & $100.00 \mathrm{a}$ & $100.00 \mathrm{a}$ \\
\hline Diu+Hex+Sulfom & $74.50 \mathrm{a}$ & $99.58 \mathrm{a}$ & $100.00 \mathrm{a}$ & $100.00 \mathrm{a}$ \\
\hline Diu+Hex+Sulfom & $71.25 \mathrm{a}$ & $92.08 \mathrm{a}$ & $100.00 \mathrm{a}$ & $100.00 \mathrm{a}$ \\
\hline Amicarbazone & $86.83 \mathrm{a}$ & $99.83 \mathrm{a}$ & $100.00 \mathrm{a}$ & $100.00 \mathrm{a}$ \\
\hline Amicarb+Isoxafl & $77.50 \mathrm{a}$ & $100.00 \mathrm{a}$ & $99.17 \mathrm{a}$ & $97.08 \mathrm{a}$ \\
\hline $\mathrm{CV}(\%)$ & 21.10 & 10.92 & 1.02 & 2.13 \\
\hline F treat. & $1.74^{\mathrm{ns}}$ & $0.98^{\mathrm{ns}}$ & $1.40^{\mathrm{ns}}$ & $3.77^{\mathrm{ns}}$ \\
\hline F drought & $9.60 * *$ & $0.97^{\mathrm{ns}}$ & $1.22^{\mathrm{ns}}$ & $2.54^{\mathrm{ns}}$ \\
\hline F treat.xdrought & $1.54^{\mathrm{ns}}$ & $0.96^{\mathrm{ns}}$ & $0.69^{\mathrm{ns}}$ & $2.54 * *$ \\
\hline LSD treat. & 19.77 & 13.01 & 1.24 & 2.57 \\
\hline LSD drought & 14.48 & 9.53 & 0.91 & 1.89 \\
\hline
\end{tabular}

** Significant at $\alpha=0.01$. ns Not significant at $\alpha=0.05 .{ }^{1 /}$ Within a column, means followed by the same letter are not significantly different according to Tukey's Multiple Mean Comparison test.

Table 6 - Visual assessment of $I$. hederifolia control as a function of days of water restriction after application (DWR) and chemical treatments. Visual evaluation was carried out at four different days after the restoration of moisture (DARM)

\begin{tabular}{|c|c|c|c|c|c|c|c|c|c|}
\hline \multirow{2}{*}{ Chemical treatment } & \multirow{2}{*}{ ose $\left(\mathrm{g} \mathrm{ha}^{-1}\right)$} & \multicolumn{4}{|c|}{ Chemical control of IPOHF at 7 DARM } & \multicolumn{4}{|c|}{ Chemical control of IPOHF at 14 DARM } \\
\hline & & 0 DWR & $30 \mathrm{DWR}$ & 60 DWR & 90 DWR & 0 DWR & $30 \mathrm{DWR}$ & $60 \mathrm{DWR}$ & 90 DWR \\
\hline Diu+Hex+suirom & 35 & $91.67 \mathrm{Aa}^{1 /}$ & 66.6 & $\mathrm{Aa}$ & $\mathrm{Aa}$ & $3 \mathrm{Aa}$ & $7 \mathrm{Aa}$ & $100.00 \mathrm{Aa}$ & $9 \mathrm{Aa}$ \\
\hline $\mathrm{Diu}+\mathrm{H}$ & $507,5+$ & $0.00 \mathrm{ABb}$ & $60.00 \mathrm{Ab}$ & $70.00 \mathrm{Aab}$ & $97.00 \mathrm{Aa}$ & $100.00 \mathrm{Aa}$ & $98.00 \mathrm{Aa}$ & $100.00 \mathrm{Aa}$ & $0 \mathrm{Aa}$ \\
\hline $\mathrm{Diu}+\mathrm{H}$ & $58,25+46$ & $55.00 \mathrm{ABb}$ & $60.00 \mathrm{Ab}$ & $85.00 \mathrm{Aab}$ & $98.00 \mathrm{Ab}$ & $100.00 \mathrm{Aa}$ & $98.33 \mathrm{Aa}$ & $100.00 \mathrm{Aa}$ & $100.00 \mathrm{Aa}$ \\
\hline Diu+Hex+Sulfom & $09+510+43,5$ & $63.33 \mathrm{ABa}$ & $60.00 \mathrm{Aa}$ & $77.67 \mathrm{Aa}$ & $83.50 \mathrm{Aa}$ & $70.00 \mathrm{Bb}$ & $98.33 \mathrm{Aa}$ & $100.00 \mathrm{Aa}$ & $100.00 \mathrm{Aa}$ \\
\hline Ami & & $\mathrm{Ba}$ & & & & $\mathrm{Aa}$ & & $\mathrm{Aa}$ & $0 \mathrm{Aa}$ \\
\hline$\Delta \mathrm{n}$ & 10,0 & $46.67 \mathrm{Bb}$ & $93.33 \mathrm{Aa}$ & $83.33 \mathrm{Aa}$ & $86.67 \mathrm{Aa}$ & $10.000 \mathrm{Aa}$ & $100.00 \mathrm{Aa}$ & $100.00 \mathrm{Aa}$ & $100.00 \mathrm{Aa}$ \\
\hline \multirow{2}{*}{ Chemical treatment } & \multirow{2}{*}{ ose $\left(\mathrm{g} \mathrm{ha}^{-1}\right)$} & \multicolumn{4}{|c|}{ Chemical control of IPOHF at 21 DARM } & \multicolumn{4}{|c|}{ Chemical control of IPOHF at 28 DARM } \\
\hline & & 0 DWR & $30 \mathrm{D}$ & $60 \mathrm{DWR}$ & 90 DWR & 0 DWR & $30 \mathrm{DWR}$ & $60 \mathrm{DWR}$ & $90 \mathrm{DWR}$ \\
\hline 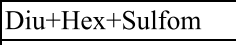 & $381+391+33,35$ & $98.33 \mathrm{Aa}$ & $\mathrm{Aa}$ & 100. & $100.00 \mathrm{Aa}$ & $100.00 \mathrm{Aa}$ & $100.00 \mathrm{Aa}$ & $100.00 \mathrm{Aa}$ & $100.00 \mathrm{Aa}$ \\
\hline Diu+Hex+Sulfom & $1507,5+425+36,25$ & $100.00 \mathrm{Aa}$ & $100.00 \mathrm{Aa}$ & $100.00 \mathrm{Aa}$ & $100.00 \mathrm{Aa}$ & $100.00 \mathrm{Aa}$ & $100.00 \mathrm{Aa}$ & $100.00 \mathrm{Aa}$ & $100.00 \mathrm{Aa}$ \\
\hline $\mathrm{Diu}+\mathrm{Hex}+$ Sulfom & $658,25+467,5+39,87$ & $100.00 \mathrm{Aa}$ & $100.00 \mathrm{Aa}$ & $100.00 \mathrm{Aa}$ & $100.00 \mathrm{Aa}$ & $100.00 \mathrm{Aa}$ & $100.00 \mathrm{Aa}$ & $100.00 \mathrm{Aa}$ & $100.00 \mathrm{Aa}$ \\
\hline Diu + Hex + Sulfom & $1809+510+43,5$ & $100.00 \mathrm{Aa}$ & $100.00 \mathrm{Aa}$ & $100.00 \mathrm{Aa}$ & $100.00 \mathrm{Aa}$ & $100.00 \mathrm{Aa}$ & $100.00 \mathrm{Aa}$ & $100.00 \mathrm{Aa}$ & $100.00 \mathrm{Aa}$ \\
\hline Amicarbazone & 1190 & $100.00 \mathrm{Aa}$ & $100.00 \mathrm{Aa}$ & $100.00 \mathrm{Aa}$ & $100.00 \mathrm{Aa}$ & $100.00 \mathrm{Aa}$ & $100.00 \mathrm{Aa}$ & $100.00 \mathrm{Aa}$ & $100.00 \mathrm{Aa}$ \\
\hline Amicarb+Isoxafl & $840+82,5$ & $98.33 \mathrm{Aa}$ & $100.00 \mathrm{Aa}$ & $100.00 \mathrm{Aa}$ & $100.00 \mathrm{Aa}$ & $100.00 \mathrm{Aa}$ & $100.00 \mathrm{Aa}$ & $90.00 \mathrm{Bb}$ & $98.33 \mathrm{Aa}$ \\
\hline
\end{tabular}

${ }_{1}^{1 /}$ Means within a column followed by the same uppercase letter or means within a row followed by the same lowercase letter are not significantly different according to Tukey's F-protected test at $\mathrm{P}=0.05$.

chemical treatment, which resulted in dry mass reduction. The four diuron + hexazinone + sulfometuron-methyl doses have reduced I. hederifolia dry mass to zero, whereas treatments with amicarbazone have not.
Chemical treatments reduced I. hederifolia dry mass at all DWR periods, and reached zero for 0, 30 and 60 DWR (Table 8). Amicarbazone and amicarbazone + isoxaflutole, however, did not reach the same effectiveness after 90 DWR, 
and diuron + hexazinone + sulfometuronmethyl maintained its effectiveness.

Although Garret \& Orson (1989) have reported that weed control with pre-emergence herbicides at dry soils is not as effective as the post-emergence ones, our study shows that diuron + hexazinone + sulfometuron-methyl,

Table 7 - I. hederifolia dry mass average as a function of days of water restriction after application (DWR) and chemical treatments. This evaluation was conducted 28 days after the restoration of moisture (DARM)

\begin{tabular}{|l|c|c|}
\hline & Days of water restriction & $\begin{array}{c}\text { IPOHF dry mass } \\
(\mathrm{g})\end{array}$ \\
\hline & 0 & $0.1146 \mathrm{a}^{\mathbf{1}^{\prime}}$ \\
\hline & 30 & $0.1549 \mathrm{a}$ \\
\hline & 60 & $0.3000 \mathrm{a}$ \\
\hline Chemical treatment & \multicolumn{1}{|c|}{${\text { Dose }\left(\mathrm{g} \mathrm{ha}^{-1}\right)}^{-1}$} & $0.2522 \mathrm{a}$ \\
\hline Diu+Hex+Sulfom & $387+391+33,35$ & $0.0000 \mathrm{~b}$ \\
\hline Diu+Hex+Sulfom & $1507,5+425+36,25$ & $0.0000 \mathrm{~b}$ \\
\hline Diu+Hex+Sulfom & $1658,25+467,5+39,87$ & $0.0000 \mathrm{~b}$ \\
\hline Diu+Hex+Sulfom & $1809+510+43,5$ & $0.0000 \mathrm{~b}$ \\
\hline Amicarbazone & \multicolumn{1}{|c|}{1190} & $0.0292 \mathrm{~b}$ \\
\hline Amicarb+Isoxafl & $840+82,5$ & $0.0083 \mathrm{~b}$ \\
\hline Control & & $1.4005 \mathrm{a}$ \\
\hline & CV(\%) & 135.97 \\
\hline & F treat. & $42.73^{* *}$ \\
\hline & F drought & $1.97^{\text {ns }}$ \\
\hline & F treat.xdrought & $1.82^{*}$ \\
\hline & LSD treat. & 0.3488 \\
\hline & LSD drought & 0.2283 \\
\hline
\end{tabular}

*, ** Significant at $\alpha=0.05$ and 0.01 , respectively. ${ }^{\text {ns }}$ Not significant at $\mathrm{a}=0.05 .{ }^{1 /}$ Within a column, means followed by the same letter are not significantly different according to Tukey's Multiple Mean Comparison test. amicarbazone and amicarbazone + isoxaflutole are effective for morning glory control, even after 60 DWR. However, only diuron + hexazinone + sulfometuron-methyl is able to fully control these broad-leaved weeds, in a broad dose range.

Once the persistence of herbicides in soil varies according to soil type (Silva et al., 1999), maybe the great efficacy of diuron + hexazinone + sulfometuron-methyl is partly due to the clay soil used in this study. Clay soils generally increase the portion of adsorbed herbicide, causing it to remain in the soil longer than usual (Sherburne \& Freed, 1954). Isoxaflutole applied with amicarbazone did not increase morning glory control compared with amicarbazone applied alone. Its low solubility in water is an unwanted herbicide feature for water restriction condition. Although isoxaflutole is stable under drought with potential residual effects for more than 60 days (Beltran et al., 2001; Rodrigues \& Almeida, 2011), our results shows that it was not effective at 90 DWR.

Diuron has intermediate water solubility (Hornsby et al., 1990), thus, it might not be associated with the effectiveness of diuron + hexazinone + sulfometuron-methyl. However, the degradation of phenylurea herbicides in nature can be a relatively slow process (Liu, 2010), and this is very relevant to justify our results.

Although the decomposition rate of the active ingredient sulfometuron-methyl is rapid under field conditions (Zahnow, 1985), it

Table 8 - I. hederifolia dry mass as a function of days of water restriction after application (DWR) and chemical treatments. This evaluation was conducted 28 days after the restoration of moisture (DARM)

\begin{tabular}{|l|c|c|c|c|c|}
\hline \multirow{2}{*}{ Chemical treatment } & \multirow{2}{*}{ Dose $\left(\mathrm{g} \mathrm{ha}^{-1}\right)$} & \multicolumn{4}{c|}{ IPOHF dry mass (g) } \\
\cline { 3 - 6 } & & $0 \mathrm{DWR}$ & $30 \mathrm{DWR}$ & $60 \mathrm{DWR}$ & $90 \mathrm{DWR}$ \\
\hline Diu+Hex+Sulfom & $387+391+33,35$ & $0.0000 \mathrm{Ba}^{1 /}$ & $0.0000 \mathrm{Ba}$ & $0.0000 \mathrm{Ba}$ & $0.0000 \mathrm{Ba}$ \\
\hline Diu+Hex+Sulfom & $1507,5+425+36,25$ & $0.0000 \mathrm{Ba}$ & $0.0000 \mathrm{Ba}$ & $0.0000 \mathrm{Ba}$ & $0.0000 \mathrm{Ba}$ \\
\hline Diu+Hex+Sulfom & $1658,25+467,5+39,87$ & $0.0000 \mathrm{Ba}$ & $0.0000 \mathrm{Ba}$ & $0.0000 \mathrm{Ba}$ & $0.0000 \mathrm{Ba}$ \\
\hline Diu+Hex+Sulfom & $1809+510+43,5$ & $0.0000 \mathrm{Ba}$ & $0.0000 \mathrm{Ba}$ & $0.0000 \mathrm{Ba}$ & $0.0000 \mathrm{Ba}$ \\
\hline Amicarbazone & 1190 & $0.0000 \mathrm{Ba}$ & $0.0000 \mathrm{Ba}$ & $0.0000 \mathrm{Ba}$ & $0.1170 \mathrm{Ba}$ \\
\hline Amicarb+Isoxafl & $840+82,5$ & $0.0000 \mathrm{Ba}$ & $0.0000 \mathrm{Ba}$ & $0.0000 \mathrm{Ba}$ & $0.0331 \mathrm{Ba}$ \\
\hline Control & - & $0.8022 \mathrm{Ac}$ & $1.0842 \mathrm{Abc}$ & $2.1003 \mathrm{Aa}$ & $1.6152 \mathrm{Aab}$ \\
\hline
\end{tabular}

$1 /$ Means within a column followed by the same uppercase letter or means within a row followed by the same lowercase letter are not significantly different according to Tukey's F-protected test at $\mathrm{P}=0.05$. 
is slightly soluble in water (Hornsby et al., 1990). These features are not able to explain the diuron + hexazinone + sulfometuronmethyl excellence in weed control observed after 90 DWR; thus sulfometuron-methyl had to be used with the other active ingredients.

According to Roy et al. (1989), the time required for hexazinone residue to remain at $50 \%$ can be 43 days in clay soils (Roy et al., 1989). Therefore, only hexazinone subdoses may have remained after 90 days of hexazinone application in our assay. Nevertheless, the mixture used in this experiment with diuron and sulfometuron-methyl showed high weed control.

Despite the unclear issues mentioned, regarding each active ingredient, diuron + hexazinone + sulfometuron-methyl may have been effective due to synergism (Hydrik \& Shaw, 1994). In conclusion, the most effective treatment for the control of morning glories (I. grandifolia and I. hederifolia) is the diuron + hexazinone + sulfometuron-methyl mixture, at the doses of $1387+391+33.35,1507.5+$ $425+36.25,1658.25+467.5+39.87$ and 1809 $+510+43.5$ g a.i. ha ${ }^{-1}$.

\section{LITERATURE CITED}

BELTRAN, E. et al. Kinetics of chemical degradation of isoxaflutole: influence of the nature of aqueous buffers (alkanoic acid/sodium salt vs phosphate). Pestic. Manag. Sci., v. 57, n. 4, p. 366-371, 2001.

CHITSAZ, M.; NELSON, D. C. Comparison of various weed control programs for potatoes. Am. Potato J., v. 60, n. 4, p. 271-280, 1983.

DAYAN, F. E.; TRINDADE, M. L. B.; VELINI, E. D. Amicarbazone, a New Photosystem II Inhibitor. Weed Sci., v. 57, n. 6, p. $579-583,2009$.

EASTMAN, D. G.; COBLE, H. D. Differences in the control of five morning-glory species by selected soybean herbicides. Proc. Southern Weed Sci. Soc., v. 30, n. 1, p. 30-45, 1977.

GARRETT, H. J.; ORSON, J. H. Depth and date of emergence of volunteer oilseed rape (Brassica napus L.) and its control with herbicides used in peas, beans, potatoes and sugar beet. Proceedings... British Crop Protection Conference, Weeds, UK, v. 3, p. 811-816, 1989.

HORNSBY A. G. et al. Managing pesticides for crop production and water quality protection. Florida Grower Rancher, v. 83, n. 1, p. 34-38, 1990.

Planta Daninha, Viçosa-MG, v. 32, n. 2, p. 345-353, 2014
HYDRICK D. E.; SHAW, D. R. Effects of tank-mix combinations of non-selective foliar and selective soil-applied herbicides on three weed species. Weed Technol., v. 8, n. 1, p. 129-133, 1994.

LIU, J. Phenylurea herbicides. In: KRIEGER, R. (Ed.). Hayes' handbook of pesticide toxicology. 3.ed. London: Academic Press, 2010. p. 1725-1731.

LUSCOMBE, B. M. et al. A novel herbicide for broad leaf and grass weed control in maize and sugar cane. In: BRIGHTON CROP PROTECTION CONFERENCE WEEDS; 1., 1985. Crop protection conference, 1985. p. 35-42.

MARTINI, G.; DURIGAN, J. C. Influência do teor de água na superfície do solo sobre a eficácia e seletividade do flazasulfuron, na cultura de cana-de-açúcar. Planta Daninha, v. 22 , n. 2 , p. $259-267,2004$

NEGRISOLI, E. et al. Influência da palha e da simulação de chuva sobre a eficácia da mistura formulada clomazone + hexazinone no controle de plantas daninhas em área de canacrua. Planta Daninha, v. 29, n. 1, p. 169-177, 2011.

PALLET, K. E. et al. The mode of action of isoxaflutole. I. Physiological effects, metabolism, and selectivity. Pestic. Biochem. Physiol., v. 62, n. 1, p. 113-124, 1998.

PETERSON, H. G. et al. Toxicity of hexazinone and diquat to green algae, diatoms, cyanobacteria and duckweed. Aquatic Toxicol., v. 39, n. 2, p. 111-134, 1997.

RODRIGUES, B. N.; ALMEIDA, F. S. Guia de herbicidas. 6.ed. Londrina: 2011. 697 p.

ROY, D. N. et al. Determination of persistence, movement, and degradation of hexazinone in selected Canadian boreal forest soils. J. Agric. Food Chem., v. 37, n. 2, p. 443-447, 1989.

SCHROEDER, W. R. planting and establishment of shelterbelts in humid severe-winter regions. Agric. Ecosyst. Environ., v. 22/23, p. 441-463, 1988.

SHERBURNE, H. R.; FREED, V. H. Soil effects on herbicides, adsorption of 3(p-chlorophenyl)-1,1-dimethylurea as a function of soil constituents. J. Agric. Food Chem., v. 2, n. 18, p. 937-939, 1954.

SILVA, A. A. et al. Controle de plantas daninhas. Brasília: Associação Brasileira de Educação Agrícola Superior; Viçosa, MG: Universidade Federal de Viçosa, 1999. 260 p.

THAKAR, C.; SINGH, H. N. Nilkalamine (Ipomoea hederacea), a menace to sugar cane. Hortic. Abstr., v. 24, n. 1, p. $530,1954$. 
TOLEDO, R. E. B. et al. Dinamic (amicarbazone), novo herbicida seletivo para o controle de plantas daninhas em pré e pós emergência na cultura da cana-de-açúcar. In: CONGRESSO BRASILEIRO DA CIÊNCIA DAS PLANTAS DANINHAS, 2004. Resumos... São Pedro: 2004. 245 p.

TOLEDO, R. E. B. et al. Manejo de corda-de-viola (Ipomoea grandifolia) no sistema de cana-crua com herbicidas Front, Velpar K e associações de Velpar K e outros herbicidas. In: CONGRESSO BRASILEIRO DA CIÊNCIA DAS

PLANTAS DANINHAS, 27., 2010. Anais... Ribeirão Preto: 2010. p. 2407-2409.
WEHTJE, G. et al. Sorption and mobility of sulfometuron and imazapyr in five alabama soils. Weed Sci., v. 35, n. 6, p. 858-864, 1987.

YONCE, H. D.; PALMER, J. H. Evaluation of method, rate, and time of application of bentazon for cocklebur control in soybeans. Proc. Southern Weed Sci. Soc., v. 29, n. 1, p. 104, 1976.

ZAHNOW, W. E. Analysis of the herbicide sulfometuron methyl in soil and water by liquid chromatography. J. Agric. Food Chem., v. 33, n. 3, p. 479-483, 1985. 